\title{
Factores de riesgo para el desarrollo psicomotor del lactante de nivel socioeconómico bajo
}

\author{
María Isabel Lira L. ${ }^{1}$ \\ Risk factors for psychomotor development in infants from \\ low socioeconomic level
}

\begin{abstract}
The purpose of this study was to assess it cerlain porent and child characteristics, which are usually registered in the child's clinical records, could be considered as risk faclors for psychomotor development in a sample of 943 infanis. The selecked variables were: children's birth weight and craneal circumference, parental age, educalion and occupalion. For those variables significantly associaied with the development quotient [DQ;, adds ratio were os follows: low birith weight (10.49). lather or mother with 5 or less years of education (14.03 and 7.09 ) and mother being older than 40 years [4.42). The orher varlables hod lower scores and were dependent from the former. Sensibitity of the four mentioned faclors was low $\{53,39,35$ and 9 ) and specificily was high (between 93 and 98 . Populalion attributable risks ranged from 48.9 llow birth weight) to 5.5 (mother older than 40 years].
\end{abstract}

[Key words: Fsychomotor developemenl, birth weight, head circumference, parenlal formal education, parental age.]

La proporción de nínos con desarrollo psicomotor subnormal es mayor entre los que crecen en scctores pobres, ${ }^{1,2}$. El nivel sociocconómico bajo sería un factor de riesgo, pero por ser una condición muy heterogénea y afectar a un porcentaje significativo de la población, no permite por sí sola focalizar la atención de salud. Si bien la proporción de niños subnormales es mayor en la población de nivel socioeconómico (NSE) bajo que en la de su homónimo medio-alto, muchos niffos que crecen en condiciones precarias se dcsarrollan normalmente. Se requiere, por lo tanto, desagregar las variables que componen un ambiente pobre e individualizar las que estarían más específicamente asociadas a retrasos del desarrollo psíquico.

En psicología evolutiva se han acumulado conocimientos sobre las influencias ambientales positivas y negalivas para el desarrollo infantil, de manera que, teóricamente, no sería dificil explicar por qué en los sectores pobres cl desarrollo

1. Psicóloga, Magíster en Salud Pública. Centro de Estudios de Desamollo y Estimulación Psicosocial (CEDEP). Investigación financiada en parte por el Fondo Nacional de Desarrollo Científico y 'Tecnológico. Proyecros 01d/ 90 y $062 / 91$. psiquico se ve más amenazado ${ }^{3}$. Por ejemplo, el desarrollo emocional y social del niño surge de la relación que establece con las personas que lo cuidar. La calidad de este vínculo depende del grado dc aceptación con que el adulto recibe al niño, su sensibilidad o intuición para captar lo que éste expresa, su destreza para interpretar correctamente lo que desea y la posibilidad de responder en forma oportuna. El vínculo con cuidadores estables genera un sentimiento de bienestar y confianza básica ${ }^{4}$ y es fundamental no sólo para el desarrollo socioemocional sino también para el cognitivo. Resulta fácil comprender que cuando una familia debe invertir todo su tiempo en luchar por sobrevivir, el esfuerzo y las tensiones consumen la energía que se requiere para criar al niño con calidez y hacen más difícil generar una interacción positiva. Si la ausencia o fragilidad del vínculo del lactante con sus cuidadores constituye un factor de ricsgo para su desarrollo psíquico, lo lógico sería identificar a los niños que estén en esa situación para enfocar hacia ellos mayor atención. No obstante variables bien identificadas, conceptualizadas e investigadas, no ha sido posible aún poner en operacion un sistema que permita evaluar su presencia mediante indicadores válidos, confiables y suficientemente simples 5 . 
Para crecer sano psíquicamente, el niño necesita, además del vinculo con sus cuidadores, posibilidades de intercambio activo con su medio, oportunidades para explorar, conocer personas, objetos y situaciones diversas, ensayar, cometer errores y aprender de ellos. Cuando la vivienda es pequefia, existe hacinamiento y no se dispone de tiempo para vigilar el juego de los niños, las posibilidades favorables a un intercambio activo con el medio se reducen drásticamente. La calidad del ambiente de juego ha sido también ampliamente estudiada ${ }^{3}$, pero al igual que con el vínculo, los procedimientos de evaluación utilizados han sido diseñados para investigaciones, empleando técnicas complejas y costosas en la perspectiva de programas de salud, por ejemplo observación en el hogar y entrevistas ${ }^{6}$. Mientras se identifican variables específicas responsables de retrasos en el desarrollo y éstas se traducen en indicadores prácticos, es urgente definir criterios para enfocar más acertadamente la atención en los niños de NSE bajo o a qué aspectos de la prevención conviene dar prioridades para favorecer el desarrollo infantil mientras se corrige la situación de los pobres.

En las fichas clínicas de los niños que concurren a los consultorios del servicio de salud, los que en medida importante son de NSE bajo, sc registran habitualmente algunos indicadores familiares y personales. El propósito de este análisis fue evaluar en qué medida ellos se asociaban al desarrollo psicomotor de los niños. A partir de esa información sería posible enfocar la atención en los niffos expuestos a factores que impliquen mayor riesgo.

\section{Método}

Se evaluó el desartolla psicomotor de niños de 4,8,12, 15,18 y 21 meses que concurrían a control del niño sano en el Consultorio Lucas Sierra (SSMN), mediante una escala de evaluación del desarrollo psicomotor que otorga a cada examinado una calificación estándar, denominada coeficiente de desarrollo (CD), que puede interpretarse como sigue:

CD > 84: normal (menor a $1 \mathrm{DE}$ bajo el promedio). CD 70 a 84: alena (entre 1 y 2 DE bajo el promedio). CD < 70 : retraso (inferiores $22 \mathrm{DE}$ bajo el promedio) ${ }^{7}$.

Se intento reunir 30 o más niños en cada categoría de desarrollo (nomal, alents y retraso) de cada grupo etáreo. Para aumenur las posibilidades de encontrar casos con CD bajo, se evalúó también a todos los rúnos de las edades mencionadas que estaban intemados en centros de la Corporación para la Nutrición Infantil (CONDN) de Santiago. No se evaluaron sujetos cuyas curacterísticas físicas corres- pondían a afecciones asociadas a sctardos del desarrollo psíquico, por ejemplo: síndrome de Down, microcefalia, parálisis cerebral. Todas las evaluaciones fueron hechas por la autora, guien tiene amplia experiencia en el uso de la técnica. La muestra quedó constituida como se indica en la tabla 1 .

En cada caso evaluado obtuvo la infornación registra. da en su ficha relativa a: peso y perímetro craneano al nacer, edad, años de estudio (escolar) aprobados y ocupación de ambos padres al nacimiento del niño (variables independientes oposibles factores de riesgo). Los dalos fuejon digitados dos veces en computador para detectar errores, verificándose adernás los valores fuera de márgenes y los cálculos que se habian efectuado manualmente para la deteminación de la edad mental.

Se estudió la correlación entre las variables independientes y el CD (chi cuadrado). Se calculó el riesgo relativo estimado de las variables que mostraron asociación significativa. Se evaluó la posible dependencia entre los factores de riesgo y -para los cuatro indicadores seleccionados- se calculo la sensibilidad, especificidad y riesgo atribuible poblacional.

\section{Resultados}

A continuación se presentan las asociaciones obscrvadas entre las variables elegidas y el CD registrado. Los cálculos se hicieron por separado para el primer y segundo año de vida, considerando que el efecto negativo de algunas variables podria cambiar con la edad ${ }^{\$ .9}$. Los resultados obtenidos se presentan en la tabla 2. La ocupación de la madre se registró, pero no se incluyó en los análisis, porque más de $90 \%$ de los casos se concentraba en la categoría "duefia de casa". Es posible que la información anotada en la ficha del niño no correspondiese a la ocupación habitual sino a la que la madre desempenaba en el momento del nacimiento del niřo. La mayoría de

Tabla 1

Composición de la muestra según la edad y coeficientes de desarrollo

\begin{tabular}{|c|c|c|c|c|}
\hline \multirow{2}{*}{$\begin{array}{l}\text { Edad del nlño } \\
\text { al momento } \\
\text { de la evaluación }\end{array}$} & \multicolumn{3}{|c|}{ Coeficientes de desarrollo } & \multirow[t]{2}{*}{ Total } \\
\hline & $<70$ & $70-84$ & $>85$ & \\
\hline 4 meses & 12 & 43 & 72 & 127 \\
\hline 8 meses & 43 & 27 & 103 & 173 \\
\hline 12 meses & 33 & 31 & 96 & 160 \\
\hline 15 meses & 31 & 35 & 94 & 160 \\
\hline 18 meses & 30 & 29 & 120 & 179 \\
\hline 21 meses & 30 & 44 & 70 & 144 \\
\hline Toul & 179 & 209 & 555 & 943 \\
\hline
\end{tabular}


Tabla 2

Asociaciones entre algunas variables y el coeficiente de desarrollo durantc el primer y segundo año de vida

\begin{tabular}{|c|c|c|c|c|c|c|c|c|}
\hline \multirow[b]{2}{*}{ Varlable } & \multirow[b]{2}{*}{$\mathbf{n}$} & \multicolumn{2}{|c|}{ Primer año } & \multirow[b]{2}{*}{$\mathbf{p} \leq$} & \multirow[b]{2}{*}{$\mathbf{n}$} & \multicolumn{2}{|c|}{ Sequndo año } & \multirow[b]{2}{*}{$\mathbf{p} \leq$} \\
\hline & & $x^{2}$ & gl & & & $x^{2}$ & gI & \\
\hline Peso nacimiento & 447 & 113,204 & 4 & 0,01 & 436 & 52,553 & 4 & 0,01 \\
\hline Perímetro craneano & 323 & 26,550 & 4 & 0,01 & 313 & 7,197 & 4 & $\mathrm{n}$ \\
\hline Edad madre & 438 & 17,576 & 6 & 0,01 & 427 & 9,523 & 6 & $\mathrm{~ns}$ \\
\hline Edad padre & 408 & 14,703 & 6 & 0,05 & 397 & 25,028 & 6 & 0,01 \\
\hline Escolaridsd de la madre & 393 & 22,654 & 4 & 0,01 & 398 & 42,841 & 4 & 0,01 \\
\hline Escolaridad del padre & 334 & 31.376 & 4 & 0.01 & 356 & 52,632 & 4 & 0,01 \\
\hline Ocupación del padre & 318 & 11,467 & 6 & ns & 340 & 11,900 & 6 & $\mathrm{~ns}$ \\
\hline
\end{tabular}

las variables escogidas se correlacionaron significativamente con el $\mathrm{CD}$, con excepción de la ocupación del padre -que no muestra asociación ni el primer ni el segundo ano de vida-, el perímetro craneano y la edad materna -que muestran una asociación significativa con el CD sólo en el primer año de vida-.

El peso de nacimiento era $\leq 2500 \mathrm{~g}$ en $43 \%$ de los niños con retraso del desarrollo psicomotor durante el primer año de vida y sólo en $3 \%$ de los normales. Algo similar se dio en el segundo año de vida, observándose en $40 \%$ de los primeros y $8 \%$ de los que no registraban retraso psicomotor.

Entre los niños con retraso durante el primer año de vida, $43 \%$ había tenido perímetro craneano $\leq 33 \mathrm{~cm}$ al nacer, hecho que sc registraba en sólo $16 \%$ de los normales. En el segundo año de vida las diferencias entre los niños con y sin retardo fueron menores para este antecedente: $42 \%$ y $28 \%$ respectivamente.

En los niños con retraso durante el primer año de vida eran más frecuentes las edades maternas $\geq 40$ años $\mathrm{y} \leq 19$ años. En el segundo año de vida la diferencia se registró sólo en frecuencia de madres $\geq 40$ años ( $5 \%$ de los niños con retardo y sólo $1 \%$ de los normales).

Entre las madres de los niños con retraso psicomotor durante el primer año de vida, se observó mayor porcentaje de baja escolaridad ( $\$ 4$ años de enseñanza básica): $24 \%$ vs. $6 \%$ en las de lactantes normales. Esta situación se invierte para la categoría de escolaridad más alta $\geq 9$ años): sólo $36 \%$ de los ninos con CD bajo, ante $63 \%$ entre los normales. Entre los nintos mayores de un año se mantienen las relaciones enunciadas $-35 \%$ y $73 \%$ respectivamente-
La asociación del CD con la edad del padre en los ninos evaluados durante el primer año de vida fue evidente sólo para padres $\geq 40$ años, quc se registraban en proporciones de $17 \%$ en niños con retraso contra $7 \%$ sin el. Esta asociación se mantuvo en los evaluados durante el segundo año de vida con $20 \%$ y $6 \%$ respectivamente. Consistentemente con lo anterior, $66 \%$ de los padres de los niños normales mayores de un año tenían entre 20 y 29 años, mientras en los niños con CD bajo esto ocurrió en $41 \%$ de los casos.

La escolaridad del padre mucstra una asociación con el CD muy similar a la observada en Ia escolaridad materna, la que se hace aún más evidente en los niños mayores de un año. En ese grupo se observa que de los niños en $C D$ bajo $27 \%$ tiene padre con menos de 5 años de escolaridad vs. 5\% en los normales. A su vez, en los niños con retardo el antecedente de padre con $\geq 9$ años de escolaridad se da sólo en $31 \%$ de los nirios en contraste con $75 \%$ de los niños normales.

La variable ocupación del padre fue clasificada en 7 categorías $^{10}$. Su asociación con el CD no fue significativa estadísticamente, pero, en las tres categorias inferiores, a mejor condición ocupacional menor porcentaje de retrasos. Esta tendencia no se mantuvo en las categorías ocupacionales superiores y fue más acentuada para los niños mayores de un año, entre los cuales los niños con retardo tenían casi el doble de padres cesantes o con ocupaciones inestables que los niños normales.

A partir de lo reseñado, aunque en algunos casos la asociación no alcanzó siempre significación estadística, se procedió a calcular para 
algunos rangos críticos de las variables, cl riesgo relativo estimado ("odds ratio" o "razón de disparidades") que corresponde a este tipo de disetto. El cálculo se hizo comparando los niffos normales (CD > 84) con los niños con retardo $(\mathrm{CD}<70$ ), excluyendo la categoría intermedia. $\mathrm{La}$ información, ordenada de mayor a menor riesgo, se presenta en la tabla 3. Antes de acometer los riesgos es preciso señalar que el análisis cualitativo de los factores permite suponer que algunos no serian independientes. Para evaluar este hecho se comparó el riesgo relativo conjunto crudo con el riesgo relativo conjunto ajustado. La información se presentra en la tabla 4.

El analisis de la dependencia existente entre las variables estudiadas permite plantear algunas posibles decisiones. Entre el peso de nacimiento y el perímetro craneano, que aparecen como dependientes, podría optarse por el peso de nacimiento, ya que el RR estimado del perímetro craneano es muy inferior $(2,10$ vs. 16,49$)$; a esto se agrega que, durante cl segundo año de vida, la asociación dcl PC al nacer con el CD no fue significativa. Las edades del padre y de la madre, como cra de esperar, no son independientes. En los mayores de 40 años podría optarse por la edad de la madre como factor de riesgo, porque éste es levemente mayor que en la edad del padre y la información está disponible con mayor frecuencia. En los menores de 20 años, el padre constituye factor de riesgo y, en el caso de la madre, el riesgo es bajo y disminuye en el segundo año de vida, razón por la cual se podría prescindir de ambas variables.

La escolaridad baja del padre y de la madre también son dependientes: el riesgo de la baja escolaridad paterna es prácticamente el doble $(14,03$ vs. 7,09). Esto no debe llamar la atención, ya que el nivel de escolaridad del padre suele afectar decisivamente el nivel socioeconómico general de la familia. No parece aconsejable, no obstante, eliminar la baja cscolaridad materna

Tabla 3

Riesgo relativo estimado de presentar retraso en el desarrollo psicomotor $(C D<70)$ en menores de dos años

\begin{tabular}{|c|c|c|c|c|c|}
\hline \multirow[t]{2}{*}{ Variable } & \multicolumn{2}{|c|}{ CD } & \multirow{2}{*}{$\begin{array}{c}\text { RR } \\
\text { Estimado }\end{array}$} & \multicolumn{2}{|c|}{ Límites de Conf. (95\%) } \\
\hline & $<70$ & $\geq 85$ & & Interior & Superior \\
\hline Peso nacimiento $<2500_{\mathrm{B}}$ & 70 & 28 & 16,49 & 15,91 & 17,09 \\
\hline Peso nacimiento $\geq 2500 \mathrm{~g}$ & 62 & 409 & & & \\
\hline Padre $<5$ A. escolaridad & 24 & 16 & 14,03 & 13,09 & 15,04 \\
\hline Padre $\geq 9$ A. escolaridad & 37 & 346 & & & \\
\hline Madre $<5$ A. escolaridad & 22 & 27 & 7,09 & 6,48 & 7,76 \\
\hline Madre $\geq 9$ A. escolaridad & 40 & 348 & & & \\
\hline Madre añosa ( > 40 años) & 9 & 8 & 4,42 & 3,33 & 5,86 \\
\hline Madre $20-29$ años & 86 & 338 & & & \\
\hline Ocupación padre categoria $1^{*}$ & 22 & 89 & 4,26 & 3,40 & 5,34 \\
\hline categoría $3^{*}$ & 8 & 138 & & & \\
\hline Padre añoso (> 40 at̃os) & 26 & 33 & 3,90 & 3,47 & 4,38 \\
\hline Padre 20 - 29 años & 61 & 302 & & & \\
\hline Madre 5-8 A. escolaridad & 51 & 137 & 3,24 & 2,97 & 3,53 \\
\hline Madre 29 A, escolaridad & 40 & 348 & & & \\
\hline Padre 5-8 A. escolaridad & 31 & 95 & 3,05 & 2,71 & 3,44 \\
\hline Padre $\geq 9$ A. escolaridad & 37 & 346 & & & \\
\hline Perímetro craneano $\leq 33$ & 33 & 92 & 2,10 & 1,73 & 2,54 \\
\hline Perímel ro craneano 34 - 35 & 35 & 205 & & & \\
\hline Ocupación padre categoría $2^{*}$ & 19 & 160 & 2,05 & 1,24 & 3,40 \\
\hline categońa $3^{*}$ & 8 & 138 & & & \\
\hline Madre adolescente ( $<20$ años) & 32 & 68 & 1,85 & 1,53 & 2,24 \\
\hline Madre 20-29 años & 86 & 338 & & & \\
\hline Padre adolescente (< 20 años) & 7 & 23 & 1,51 & 0,57 & 4,02 \\
\hline Padre 20.29 años & 61 & 302 & & & \\
\hline
\end{tabular}

- categoría 1: cesante, ocupación inestable, elc. categoría 2: jornalero, obrero no calificado, cuenta propia, etc. categoria 3: obrero calificado, chofer, elc. 


\section{Tabla 4}

Interdependencia entre los factores de riesgo

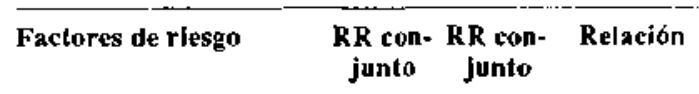

Peso de nacimiento $<2500 \mathrm{~g}$ y perímetro craneano 5,08 25,56 Dependientes Padre y madre $<20$ años $1,57 \quad 2,32$ Dependientes Padre y madre $>40$ años $\quad 4,29 \quad 10,83$ Dependientes Padre y madre $<5$ años escolaridad

$7,78 \quad 56,75$ Dependientes

Padre $<5$ años escolaridad y ocupación inestable o cesante $8,33 \quad 36,90$ Dependientes

como factor de riesgo, porque el valor que alcanzó el RR estimado de esta variable no es despreciable y cl padre no siempre está presentc.

Entre la baja escolaridad del padre y la ocupación inestable, que son dependientes, podría optarse por la escolaridad, ya que el RR estimado es mucho mayor $(14,03$ vs. 4,26$)$, la asociación de la ocupación del padre con el CD no fuc estadísticamente significativa en el primero y el segundo año de vida y la situación laboral cs más inestable.

A partir de las opciones sugeridas y con miras a la utilización de esta información en los programas de salud, podría individualizarse como factores de riesgo para el desarrollo psicosocial las siguientes variables: peso de nacimiento $<2500$ g, escolaridad del padre o de la madre inferior a 5 años y madre mayor de 40 años de edad.

Conviene conocer el grado de eficiencia que tendrian estos indicadores. Por una parte, la capacidad para identificar correctamente a aquellos que tuvieran retraso en el desarrollo (sensibilidad); y, por otra, la capacidad de identificar correctamente a los normales (especificidad). Esta información se presenta en la tabla 5. Como puede observarse, la sensibilidad de los cuatro indicadores es baja. Esto significa que si sólo se usaran ellos para enfocar la atención, un porcentaje muy importante de niños no sería detectado. El poder predictivo positivo, es decir la capacidad para predecir la presencia de retardo, fluctuaría en la población estudiada entre $45 \%$ para la escolaridad de la madrc y $71 \%$ para el peso de nacimiento. Estos valores se afectan según el porcentaje del daño en la población y son por lo tanto inferiores en la realidad, ya que debe re- cordarse que en la muestra estudiada se aumenló intencionalmente la proporción de retrasos. Se presenta también en la tabla 5 el porcentaje de niños expuestos a los cuatro riesgos. Esta información se obtuvo excluyendo los niños de CONIN, considerando sólo la muestra recogida en el Consultorio Lucas Sierra (n: 707), donde el único criterio de selección fue la edad. Debe recordarse que éste se ubica en un sector popular de la Región Metropolitana y que las cifras variarán obviamente en sectores más acomodados o más marginales. Los porcentajes de aparición de cada riesgo no pueden sumarse, ya que a veces los mismos niños están expuestos a varios de ellos.

El riesgo atribuible poblacional, que mide el descenso que ocurriría en la proporción de retrasos, si un factor fuera eliminado totalmente ${ }^{11}$, fue $48,9 \%$ para bajo peso de nacimiento; $31,9 \%$ para baja escolaridad del padre; $27,1 \%$ para baja escolaridad materna y $5,5 \%$ para edad materna superior a 40 años.

\section{Comentario}

El riesgo relativo estimado más alto de relraso psicomotor con peso de nacimiento $<2500 \mathrm{~g}$ ha de tomarse con cautela, ya que, como se sexalara al describir la muestra -para aumentar las posibilidades de contar con niños de CD bajo- se incorporo a lactantes internados cn centro de recuperación nutricional, donde la asociación cntre rctraso del desarrollo y déficit de pcso es más frecuente. Adcmás, los riesgos se midicron en función de los retrasos más severos $(C D<70)$, que suelen estar más asociados a factores biológicos que ambientales. El vator obtenido sería sin duda menor si se estudiara una población sin

Tabla 5

Características de las variables seleccionandas como indicadores de ricsgo

\begin{tabular}{lrrr} 
Factor de riesgo & $\begin{array}{c}\text { Sensi- } \\
\text { bilidad }\end{array}$ & $\begin{array}{c}\text { Especi- } \\
\text { ficidad }\end{array}$ & $\begin{array}{c}\text { \% en la } \\
\text { poblaciosi } \\
\text { extudiada }\end{array}$ \\
\hline Pcso nacimicnto $<2500 \mathrm{~g}$ & 53 & 94 & 6,2 \\
Escolaridad padre $<5$ años & 39 & 96 & 3,6 \\
Escolaridad madre $<5$ anios & 35 & 93 & 6,1 \\
Edad madre $>40$ años & 9 & 98 & $1, ?$ \\
\hline
\end{tabular}


factores nutricionales o en función de los retrasos leves (CD 70-85). A pesar de los sesgos senfalados, los resultados obtenidos concuerdan $\operatorname{con}$ otros $^{8,9}$ en atribuit al bajo peso de nacimiento un riesgo importante para el desarrollo psicomotor del lactante. De Ios cuatro factores seleccionados, éste es el más frecuente y susceptible de prevenir. Ahora bien, entre los niños con bajo peso hay prematuros, desnutridos in útero y niños con diversas afecciones. Puesto que sólo $58 \%$ de los lactantes estudiados, que tenían bajo peso al nacer, presentaron retraso psicomotor, seria importante explorar más adelante si este se asocia a un tipo especial de niños con tal antecedente, determinar si un cambio favorable entre el primero y el segundo años de vida persiste en los siguientes y comparar la evolución de estos niños en distintos niveles socioeconómicos, pues las complicaciones perinatales asociadas a retrasos del desartollo psiquico podrían tener más impacto en los menos favorecidos ${ }^{12,13}$.

Después del peso de nacimiento el riesgo relativo estimado más alto fue el nivel educacional del padre, duplicando éste al de la madre con igual escolaridad, lo que no coincide con la mayor importancia que se tiende a atribuir generalmente a las características maternas, sin considerar las paternas ${ }^{14}$. El levemente mayor impacto de la escolaridad del padre en el segundo año de vida que en el primero es comprensible, ya que los logros del desarrollo en esa edad se asocian con la exploración, descubrimiento del medio, adquisición del lenguaje, donde el nivel educacional del hogar tendría mayor importancia. Puesto que el riesgo persiste, si bien en menor escala, con padres de 5 y 8 años de escolaridad, parece haber más bien cierta continuidad, con reducción progresiva del riesgo hasta que, probablemente, la relación alcance un tope en algún punto de la ensefhanza media. El riesgo atribuible poblacional es muy semejante entre escolaridad paterna y materna, y el mayor de parte del padre se compensa con un porcentaje menor en la población estudiada. Desgraciadamente, la baja escolaridad de los padres no es fácil de eliminar, pero puede ser usada para identificar grupos en peligro. Si la escolaridad, por sí misma, fuese un contribuyente causal al retraso del desarrollo, podrian desarrollarse programas educativos que fortalecieran los conocimientos relativos a la crianza, pero, es probable que también participen otros factores, tanto en la deficiente educación como en las limitaciones para desempeñar adecuadamente su rol prenalal, por ejemplo: baja capacidad intelectual, mala autoestima ${ }_{2}$ trastornos de personalidad.

La edad extrema de los padres implica mayor riesgo para el desartollo. A diferencia de la escolaridad, las edades de los cónyuges suelen guardar fuerte paralelismo: madres jóvenes tienen con alta frecuencia parejas jóvencs y madres añosas tienen parejas mayores. La semejanza de la distribución de los datos, $y$ los efectos negativos menores en el caso del padre, sugieren que la correlación entre la edad del padre y los retrasos del desarrollo podria ser espuria. Tradicionalmente, se ha considerado de riesgo a las madres menores de 18 anos o mayores de 40 . Este cstudio sugiere que el riesgo, para el desartollo psicomotor, sería superior en los hijos de madres con más affos y aumentaría considerablemente en los hijos de éstas durante cl segundo año de vida. El estudio no permite interpretar estos resultados pero se pueden aventurar algunas hipótesis. Es posible que el grupo "menores de 20 años" sea heterogéneo; de hecho en algunos cstudios se califica distinto el riesgo en madres $<15,<17$ y $<19$ años $^{16}$. Para despejar esta duda se requeriría contar con un número mayor de casos con mađres más jóvenes. Tambiên es posible que las madres adolescentes tengan, en nuestro país, más apoyo familiar para la crianza del niño. Las madres mayores, aunque tengan más experiencia, suelen tener varios niños en esa etapa de la vida, lo que también ha sido considerado riesgo ${ }^{15}$. La edad de la madre es, en todo caso, un factor de riesgo que podría aplicarse a la prevención y tambièn para enfocar la atención, si bien. siendo baja la proporción de madres mayores de 40 antos, la eliminación de ese factor tendría un impacto muy pequeño en la disminución de los retrasos.

En síntesis, de las siete variables estudiadas, el bajo peso de nacimiento y los embarazos en madres mayores de 40 aftos serían dos aspectos a prevenir. Puesto que ambos han sido también considerados factores de riesgo para el desarrollo físico, ello no significaría incorporar acciones nuevas. Junto con la baja escolaridad de los padres, los dos factores anteriores podrían contribuir a enfocar mejor la atención hacia niños con mayor riesgo. Sin embargo, la sensibilidad de estos indicadores para pesquisar retardos existentes es relativamente baja, con proporciones de fal- 
sos positivos entre $29 \%$ y $55 \%$ y de falsos negativos entre $10 \%$ y $20 \%$ en Ios cuatro factores. Con todo, su aplicación podría ser más eficiente que limitarse a la simple evaluación de los niños. La identificación de los factores asociados al retraso del desarrollo psicomotor, de los que protcgen a ciertos niños contra sus efectos y la aplicación de métodos para controlarlos, requiere aún de muchos esfuerzos ${ }^{16-18}$.

\section{Resumen}

El propósito de este estudio fue evaluar en qué medida, características del niño y sus padres, que sc registran habitualmente en la ficha del niño, podrían considerarse indicadores de riesgo para el desarrolto psicomotor. Se seleccionaron peso de nacimiento y perímetro craneano del niño; edad, escolaridad y ocupación de ambos padres. Se estudiaron 943 lactantes. Para aquellas variables asociadas significativamente con el coeficicnte de desarrollo (CD) se calculó el riesgo relativo estimado: bajo peso de nacimiento $(16,49)$, padre o madre con 5 o menos años de escolaridad $(14,03$ y 7,09$)$ y madre mayor de 40 aflos $(4,42)$. Las demás variables tenían un ríesgo menor y eran dependientes de las anteriores. La sensibilidad de los cuatro factores senalados fue baja $(53,39,35$ y 9$)$ y la especificidad alta (entre 93 y 98). El riesgo atribuible poblacional fluctuó entre 48,9 (bajo peso de nacimiento) y 5,5 (madre mayor de 40 años).

(Palabras clave: retardo psicomotor, factores de riesgo, peso al nacer, perímetro craneal, cdad de los padres, educación de los padres.)

\section{Referencias}

1. Seguel $X$, Bralic $S$, Edwards $M$ : Más allá de la sobrevivencia. Santiago de Chile: Publicación UNICEF/ CEDEP, 1989.

2. Terro J, Piedra Cueva $E$, Roba $O$, et al.: Creciendo en condiciones de riesgo. Montevideo: Publicación UNICEF/CLAEH, 1989.
3. Myers R: Toward a fair stan for children. New York: Publicaciốn de UNESCO, 1990.

4. Erikson E: Infancia y sociedad. Buenos Aires: Ediciones Hormé, 1966.

S. Atkin L: Analysis of instruments used in Latin America to measure psychosocial development and environmental risk in children from 0-6 years of age. México: Documento del Instituto Nacional de Pcrinatología, 1989.

6. Caldwell $B$, Bradley $R$ : Home observation for the measurement of the environmerti. Arkansas: Documento de la Liniversity of Arkansas at Little Rock, 1979.

7. Rodriguez $S$, Arancibia V, Undurraga $C$ : Escala de evaluacion del desarrollo psicomolor. Buenos Aires: Editorial Galdoc, 1978.

8. Aratic $S$, Edwards $M$, Lira MI. Seguel $X$, Atalah $E$ : Proyecto colaborativo sobre estrategias de alimenación, crianza y desarrollo infantil. Santiago de Chile: Docasmento CEDEP, 1989

9. Brand $G$, Velásquez $M$, Sepútueda $D$, May $I$, Rodriguez $J$ : Estimulación temprana: diez atros de experiencia. Kev Chil Pedatr 1989; 60: 214-218.

10. ADIMARK Lida., CCC. Investigación y Gallup-Chile. Esiratificación sociceconóntca de la población del Gran Santiago. Santiago de Chile: documento mimeografiado, 1979.

11. OPS: Manual sobre el enfoque de riesgo en la atención matemoinfantil. Serie PALTEX pata ejecutores de programas de salud, 1986; $\mathrm{N}^{2} 7$.

12. Wallace IF: Socioenvitonmental issues in Jongiudinal research of high-risk infants. En: Vietze P.M. y Vaughan HG eds. Early identification of infants with developmental disabilities. Philadelphia, PA: Grune and Stratton, $1984 ; 356-375$.

13. Ayhward P: Environmental influences on the developmental outoome of children at risk. Inf Young Children 1990; $2: 1-9$.

14. Ministerio de Salud: Programa de salud del niño y del adolescente. Santiago de Chile: documento del Programa de las personas, 1981.

15. Parkyn $/ h$ : Identification of al-risk infants and preschool children. En: Frankenberg WK, Emde RN y Sullivan JW eds. Early identification of children at risk: an intemational perspective. New York: Plenum Prcss, 1985: 203-209.

16. Jens $K G$, Gordon $B N$ : Understanding risk: implications for tracking high-risk infants and making early service delivery decisions. International Journal of Disability, Development and Education, 1991; 38: 211-224.

17. King EH, Logsdon DA, Schroeder SR: Risk faclors for developmental delay among infants and toddlers. Children's Health Care, 1992; $21: 39-52$.

18. Parker S, Greer $S, Z$ Zuckerman B: Doble jeopardy: the impact of poverty on early child development. Pediatr Clin Nort Am 1988; 35: 1227.1240. 